\title{
The Leo Triplet: Common origin or late encounter? ${ }^{\star}$
}

\author{
V. L. Afanasiev ${ }^{1}$ and O. K. Sil'chenko ${ }^{2,3,4}$ \\ 1 Special Astrophysical Observatory, Nizhnij Arkhyz, 369167 Russia \\ 2 Sternberg Astronomical Institute, University av. 13, Moscow 119992, Russia \\ e-mail: olga@sai.msu.su \\ ${ }^{3}$ Isaac Newton Institute of Chile, Moscow Branch, Russia \\ ${ }^{4}$ UK Astronomy Data Centre, Guest Investigator, UK
}

Received 20 February 2004 / Accepted 27 August 2004

\begin{abstract}
The kinematics, structure, and stellar population properties in the centers of two early-type spiral galaxies of the Leo Triplet, NGC 3627 and NGC 3623, are studied by means of integral-field spectroscopy. Unlike our previous targets, NGC 3384/NGC 3368 in the Leo I Group and NGC 5574/NGC 5576 in LGG 379, NGC 3623 and NGC 3627 do not appear to experience a synchronous evolution. The mean ages of their circumnuclear stellar populations are quite different, and the magnesium overabundance of the nucleus in NGC 3627 is evidence for a very brief last star formation event 1 Gyr ago whereas the evolution of the central part of NGC 3623 looks more quiescent. In the center of NGC 3627 we observe noticeable gas radial motions, and the stars and the ionized gas in the center of NGC 3623 demonstrate more or less stable rotation. However, NGC 3623 has a chemically distinct core - a relic of a past star formation burst - which is shaped as a compact, dynamically cold stellar disk with a radius of $\sim 250-350 \mathrm{pc}$ which has been formed not later than 5 Gyr ago.
\end{abstract}

Key words. galaxies: individual: NGC 3623 - galaxies: individual: NGC 3627 - galaxies: nuclei - galaxies: stellar content galaxies: kinematics and dynamics - galaxies: evolution

\section{Introduction}

It is a long-standing problem what mechanisms define the evolution of galaxies, intrinsic or external. During the last years external mechanisms have been winning the competition: modern cosmology prescribes multiple mergers to be the main driving force of galactic evolution as regards star formation process or morphological transformations. However, intrinsic disk instabilities which may provoke a substantial secular evolution are not left out entirely. From an observational point of view one of the most promising approaches is to study in detail contemporary properties of galaxies in different environments and to search for signatures of environmental influence on these properties. Galaxy groups constitute perhaps the least-studied type of environment, opposite to clusters or pure field; meanwhile, their closeness to each other and moderate relative velocities make them very suitable objects to demonstrate the power of external mechanisms, of interactions in particular, to govern the evolution of galaxies that are members of groups. So we aim to

* Based on observations collected with the $6 \mathrm{~m}$ telescope of the Special Astrophysical Observatory (SAO) of the Russian Academy of Sciences (RAS) which is operated under the financial support of Science Department of Russia (registration number 01-43) and on data from the Isaac Newton Group (ING) and Hubble Space Telescope (HST) Archives. collect statistics on stellar population and structure properties in the central parts of early-type galaxies in nearby groups.

We choose to compare the properties of galaxies of comparable luminositites and of close morphological types, and this choice limits our sample of galaxy groups: we need at least two early-type galaxies, close to each other and bright enough, in each group. Up to now we have investigated stellar population properties, the mean (luminosity-weighted) stellar age first of all, in NGC 5576/NGC 5574 (Sil'chenko 1997; Sil'chenko et al. 2002) and in NGC 3379/NGC 3384/NGC 3368 (Sil' chenko et al. 2003). In both groups we have detected a certain coherence of star formation events in the nuclei of neighbouring galaxies. In the centers of the E-galaxy NGC 5576 and the S0-galaxy NGC 5574 the iron-to-magnesium abundance ratio exceeds the solar value and the mean age of the stellar populations is $3 \mathrm{Gyr}$. In the centers of the S0-galaxy NGC 3384 and the Sab-galaxy NGC 3368 the mean age is also found to be 3 Gyr. Moreover, in the centers of NGC 3384 and NGC 3368 we have found decoupled kinematical substructures whose spatial orientations match the orientation of the supergiant H I cloud (ring) encircling the central galaxies of the Leo I group. We have concluded that at least when a significant amount of intergalactic cool gas is available, the circumnuclear evolution of the group member galaxies have been strongly affected by their mutual interaction and/or by their interaction with intergalactic gas clouds. 
Table 1. Global parameters of the galaxies.

\begin{tabular}{|c|c|c|}
\hline NGC & 3623 & 3627 \\
\hline Type $\left(\mathrm{NED}^{1}\right)$ & $\mathrm{SAB}(\mathrm{rs}) \mathrm{a}$ & $\mathrm{SAB}(\mathrm{s}) \mathrm{b}$ \\
\hline$B_{T}^{0}\left(\mathrm{RC}^{2}\right)$ & 9.65 & 9.13 \\
\hline Distance $^{3}, \mathrm{Mpc}$ & \multicolumn{2}{|c|}{9.1} \\
\hline$M_{B}\left(\right.$ from $\left.B_{T}^{0}, \mathrm{RC} 3\right)$ & -20.15 & -20.67 \\
\hline$R_{25}, \mathrm{kpc}\left(\right.$ from $\left.D_{25}, \mathrm{RC} 3\right)$ & 12.9 & 12.1 \\
\hline$(B-V)_{T}^{0}(\mathrm{RC} 3)$ & 0.77 & 0.60 \\
\hline$(U-B)_{T}^{0}(\mathrm{RC} 3)$ & 0.32 & 0.11 \\
\hline$V_{r}, \mathrm{~km} \mathrm{~s}^{-1}(\mathrm{NED})$ & 807 & 727 \\
\hline$i_{\text {phot }}^{4}$ & $70.9^{\circ}$ & $64.2^{\circ}$ \\
\hline$P A_{\text {phot }}(\mathrm{RC} 3)$ & $174^{\circ}$ & $173^{\circ}$ \\
\hline$V_{\text {rot }}, \mathrm{km} \mathrm{s}^{-1}$, from $\mathrm{HI}^{4}$ & 266 & 202 \\
\hline$\sigma_{*}, \mathrm{~km} \mathrm{~s}^{-1}$ & $149^{5}$ & $117^{6}$ \\
\hline$M_{\mathrm{HI}}^{7}, 10^{8} M_{\odot}$ & 2.7 & 7.6 \\
\hline$M_{\mathrm{H}_{2}}{ }^{8}, 10^{8} M_{\odot}$ & 3.7 & 32.3 \\
\hline
\end{tabular}

${ }^{1}$ NASA/IPAC Extragalactic Database.

${ }^{2}$ Third Reference Catalogue of Bright Galaxies.

${ }^{3}$ Paturel et al. (2002).

${ }^{4}$ Haynes (1981).

${ }^{5}$ Whitmore et al. (1979).

${ }^{6}$ Heraudeau \& Simien (1998).

${ }^{7}$ Haynes et al. (1979).

${ }^{8}$ Sage (1993), corrected for the new distance.

In this work we consider two nearby early-type spiral galaxies belonging to the famous Leo Triplet, NGC 3623 and NGC 3627. The third member of this company, NGC 3628 , is of rather late type, and moreover, it is seen edge-on and has a dust lane completely obscuring the nucleus. The main global properties of NGC 3623 and NGC 3627 are given in Table 1 . The projected distance between the two galaxies is only $20^{\prime}$, or $\sim 50 \mathrm{kpc}$; it is comparable to the distance between our Galaxy and the LMC, and considering the existence of the Magellanic Stream, we would expect tidal interaction between NGC 3623 and NGC 3627. However these two galaxies are always mentioned as "non-interacting pair" (e.g. RC1 or RC2); instead violent interaction has been supposed to exist between NGC 3627 and NGC 3628, starting from the works of Zwicky (1956) and Vorontsov-Velyaminov (1959), though the distance between NGC 3627 and NGC 3628 is almost twice that between NGC 3627 and NGC 3623 and the difference in systemic velocities is the same (NGC 3623 and NGC 3628 have similar $v_{\text {LSR }}$ ). By inspecting either Table 1, or a DSS image of the Leo Triplet, it can be noted that NGC 3623 and NGC 3627 look almost like twins: they have the same sizes, close morphological types, close spatial orientations (their lines of nodes are parallel), the same sense of rotation with the southern parts of both receding, close luminosities, etc. NGC 3623 is a little more massive, if witness its higher rotation velocity and stellar velocity dispersion, and NGC 3627 is more gas-rich with consequently more intense global star formation and bluer integrated colours. Why don't they influence each other? Both galaxies have very similar global bars, with radii of $R \sim 2 \mathrm{kpc}$, that are aligned and so may be of tidal origin. Could we perhaps find signatures of coherent evolution in the central parts of the galaxies? Here we present results of 2D spectroscopy of the circumnuclear regions of NGC 3623 and NGC 3627. The layout of the paper is as follows. We report our observations and other data which we use in Sect. 2. The radial variations of the stellar population properties are analysed in Sect. 3, and in Sect. 4 two-dimensional velocity fields obtained by means of $2 \mathrm{D}$ spectroscopy for the central parts of NGC 3623 and NGC 3627 are presented. Section 5 gives discussion and our conclusions.

\section{Observations and data reduction}

The spectral data which we analyse in this work are obtained with two different integral-field spectrographs: the fiberlens Multi-Pupil Fiber Spectrograph (MPFS) at the $6 \mathrm{~m}$ telescope of the Special Astrophysical Observatory of the Russian Academy of Sciences (SAO RAS) and the international Tigremode SAURON at the $4.2 \mathrm{~m}$ William Herschel Telescope at La Palma.

The last modification of the MPFS became operational in the prime focus of the $6 \mathrm{~m}$ telescope in 1998 (http://www.sao.ru/hq/lsfvo/devices/mpfs/). It is a fiber-lens system: densely packed square microlenses in the focal plane of the telescope create a set of micropupils, and the fibers after them transmit the light from $16 \times 15$ square elements of the galaxy image to the slit of the spectrograph together with 16 additional fibers that transmit the sky background light taken at a distance of $4^{\prime}$ from the galaxy, so separate sky exposures are not necessary. The size of one spatial element was approximately $1^{\prime \prime} \times 1$ "; a CCD TK $1024 \times 1024$ detector was used. The reciprocal dispersion was $1.35 \AA$ per pixel, with a spectral resolution of $5 \AA$, rather stable over the field of view. The wavelength calibration was done by exposing a spectrum of a hollow cathode lamp filled with helium, neon, and argon before and after the galaxy exposures; the internal accuracy of linearization was typically $0.25 \AA$ in the green and $0.1 \AA$ in the red; additionally we checked the accuracy and the absence of a systematic velocity shift by measuring strong emission lines of the night sky [O I] $\lambda 5577$ and [O I] $\lambda 6300$. We obtained MPFS data in two spectral ranges, green, 4300-5600 , and red, 5900-7200 $\AA$. The green spectra were used to calculate the Lick indices $\mathrm{H} \beta, \mathrm{Mgb}, \mathrm{Fe} 5270$, and Fe5335 which are suitable for determining metallicity, age, and $\mathrm{Mg} / \mathrm{Fe}$ ratio of old stellar populations (Worthey 1994), and they are also used for cross-correlation with the spectrum of a template star, usually of G8III-K1III spectral type, in order to obtain the lineof-sight velocity field for the stellar component and a map of the stellar velocity dispersion. The red spectral range contains strong emission lines $\mathrm{H} \alpha$ and [N II] $\lambda 6583$ and is used to derive line-of-sight velocity fields for the ionized gas. To calibrate the new MPFS index system onto the standard Lick one, we have observed 15 stars from the list of Worthey et al. (1994) during four observational runs and calculated the linear regression formulae to transform our index measurements onto the Lick system; the rms scatters of points near the linear dependencies are about $0.2 \AA$ for all 4 indices under consideration, so they are 
Table 2. 2D spectroscopy of the galaxies studied.

\begin{tabular}{|c|c|c|c|c|c|c|}
\hline Date & Galaxy & Exposure & Configuration & Field & Spectral range & Seeing \\
\hline 9 Feb. 2000 & NGC 3623 & $45 \mathrm{~min}$ & BTA/MPFS+CCD $1024 \times 1024$ & $16^{\prime \prime} \times 15^{\prime \prime}$ & $4200-5600 \AA$ & 1.4 \\
\hline 12 Feb. 2000 & NGC 3627 & $45 \mathrm{~min}$ & BTA/MPFS+CCD $1024 \times 1024$ & $16^{\prime \prime} \times 15^{\prime \prime}$ & $4200-5600 \AA$ & 3.3 \\
\hline 14 Feb. 2000 & NGC 3623 & $52 \mathrm{~min}$ & BTA/MPFS+CCD $1024 \times 1024$ & $16^{\prime \prime} \times 15^{\prime \prime}$ & $5800-7200 \AA$ & $3 . \prime 5$ \\
\hline 15 Feb. 2000 & NGC 3627 & $30 \mathrm{~min}$ & BTA/MPFS+CCD $1024 \times 1024$ & $16^{\prime \prime} \times 15^{\prime \prime}$ & $5800-7200 \AA$ & $3 . \prime 5$ \\
\hline 29 Mar. 2000 & NGC 3623, Pos.1 & $120 \mathrm{~min}$ & WHT/SAURON+CCD $2 \mathrm{k} \times 4 \mathrm{k}$ & $33^{\prime \prime} \times 41^{\prime \prime}$ & $4800-5400 \AA$ & $1 . " 5$ \\
\hline 30 Mar. 2000 & NGC 3623 , Pos. 2 & $120 \mathrm{~min}$ & WHT/SAURON+CCD $2 \mathrm{k} \times 4 \mathrm{k}$ & $33^{\prime \prime} \times 41^{\prime \prime}$ & $4800-5400 \AA$ & $1 . " 6$ \\
\hline
\end{tabular}

within the observational errors of Worthey et al. (1994). To correct the index measurements for the stellar velocity dispersion which is usually substantially non-zero in the centers of earlytype galaxies, we have applied smoothing to the spectrum of the standard star HD 97907 by a set of Gaussians of various widths; the derived dependencies of the index corrections on $\sigma$ were approximated by polynomials of the 4 th order and applied to the measured index values before they are put onto the Lick system.

The second 2D spectrograph from which we used the data is a new instrument, SAURON, operated at the $4.2 \mathrm{~m}$ William Herschel Telescope (WHT) on La Palma - for its detailed description see Bacon et al. (2001) and for some preliminary scientific results see de Zeeuw et al. (2002). We have taken the data for NGC 3623 observed in March 2000 from the open ING Archive of the UK Astronomy Data Centre. Briefly, the field of view of this instrument is $41^{\prime \prime} \times 33^{\prime \prime}$ with a spatial element size of 0 ' $^{\prime} 94 \times 00^{\prime} 94$. The sky background is taken 2 arcmin from the center of the galaxy and is exposed simultaneously with the target. The fixed spectral range is $4800-5400 \AA$, the reciprocal dispersion is $1.11 \AA-1.21 \AA$, varying from the left to the right edge of the frame. The comparison spectrum is that of neon, and we made the linearization by fitting a polynomial of the 2 nd order with an accuracy of $0.07 \AA$. The index system is checked by using stars from the list of Worthey et al. (1994) that have been observed during the same observational run. The regressions describing the index system of the March 2000 observational run when NGC 3623 was observed can be found in our paper on the Leo I group where the SAURON data for NGC 3384 are analysed (Sil'chenko et al. 2003). The relations between instrumental and standard-system indices were approximated by linear formulae which were applied to our measurements to put them onto the standard Lick system. The stellar velocity dispersion effect was corrected in the same manner as for the MPFS data.

The full list of the exposures made for NGC 3623 and NGC 3627 with two 2D spectrographs is given in Table 2.

Additionally, to know the central structure of the galaxies, we have retrieved HST data from the HST Archive. Since both galaxies are dust-rich in the center, we try to use the reddest images available. NGC 3623 was exposed through the F814W filter in the frame of the program of S. Smartt (ID 9042) of searching progenitors of $\mathrm{SNe}$ (the nucleus of the galaxy is located in the WF3-frame, so the scale is 0 '. $^{\prime} 1$ per pixel), and NGC 3627 was observed in the frame of the programs of J. Mulchaey on the fueling of active nuclei (ID 7330) with NICMOS2 and of W. Sparks' snapshots (ID 7919) with NICMOS3. To probe the large-scale structures of the galaxies under consideration, we have also retrieved the 2MASS images from NED for both galaxies.

All the data, spectral and photometric, except the data obtained with the MPFS, have been reduced with the software produced by Dr. V. V. Vlasyuk of the Special Astrophysical Observatory (Vlasyuk 1993). Primary reduction of the data obtained with the MPFS was done in IDL with a software created by one of us (V.L.A.). The Lick indices were calculated with our own FORTRAN program as well as using the FORTRAN program of Dr. A. Vazdekis which also provides the index error calculation.

\section{Stellar population properties in the centers of NGC 3623 and NGC 3627}

Since the determination of stellar population properties requires very high accuracy of the Lick indices, we use twodimensional maps for a morphological analysis of stellar population substructures, and azimuthally averaged profiles for quantitative age and abundance estimations.

\subsection{NGC 3623}

Figure 1 presents three $16^{\prime \prime} \times 15^{\prime \prime}$ maps of the Lick indices, $\mathrm{H} \beta, \mathrm{Mgb}$, and $\langle\mathrm{Fe}\rangle \equiv(\mathrm{Fe} 5270+\mathrm{Fe} 5335) / 2$, constructed from the MPFS data, and Fig. 2 presents three $40^{\prime \prime} \times 40^{\prime \prime}$ maps of the Lick indices $\mathrm{H} \beta, \mathrm{Mgb}$, and Fe5270 constructed from the SAURON data. The latter maps are obtained by combining two independent data sets, Pos.I and Pos.II (see Table 2), which are exposures of the northern and the south-eastern parts of the galaxy, though both include the nucleus. Although the sizes of the fields of view differ greatly for the MPFS and SAURON, both Figs. 1 and 2 show the main characteristic feature of the stellar population distribution: a chemically decoupled core of NGC 3623 which looks like a resolved structure enhanced in the magnesium index and elongated northsouth. Previously Proctor et al. (2000) and Proctor (2002) reported a Mg-index peak in the nucleus of NGC 3623 from their long-slit observations along the minor axis. Proctor (2002) even gives a quantitative estimate of the metallicity drop between the nucleus, unresolved in their observations, and the bulge: from +0.6 dex to $\sim+0.1$ dex, or by a factor of 3 . But now for the first time we can establish the morphology of the 



Fig. 1. The MPFS index maps for NGC $3623 ;\langle\mathrm{Fe}\rangle \equiv(\mathrm{Fe} 5270+\mathrm{Fe} 5335) / 2$. The green $(\lambda 5000 \AA)$ continuum is overlaid by isophotes marking $10 \%, 20 \%, 30 \%$, etc., of the maximum surface brightness.
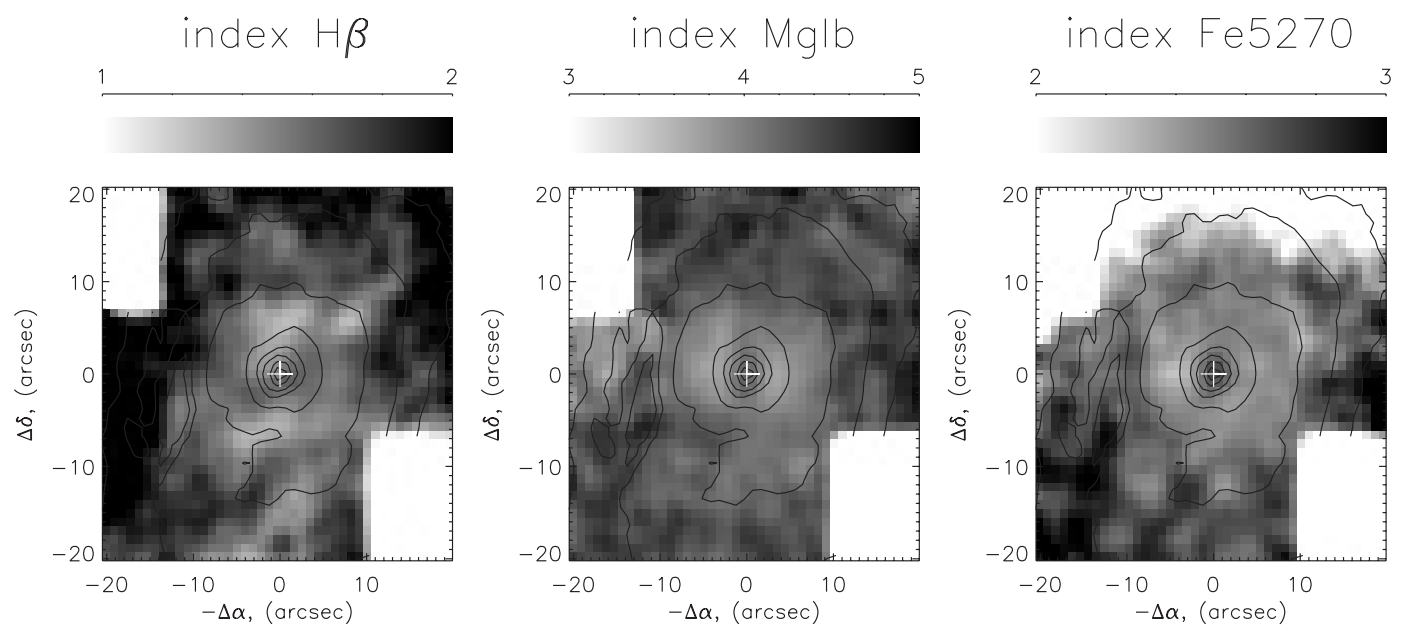

Fig. 2. The SAURON index maps for NGC 3623. The green ( $\lambda 5000 \AA$ ) continuum is overlaid by isophotes.

chemically decoupled entity: it is a circumnuclear disk or a bar with a radius of $\sim 8^{\prime \prime}(\sim 350 \mathrm{pc})$, almost aligned with the global line of nodes of the galaxy. From Fig. 1 one may get an impression that the distribution of $\langle\mathrm{Fe}\rangle$ shows a much more compact peak than that of $\mathrm{Mgb}$; it may signify that the characteristic time of secondary star formation that has produced the chemically decoupled substructure varied along the radius. The maps of Fig. 2, with their large extension, reveal two further interesting features of the index distributions: the Mgb index reaches a minimum in a shallow, almost circular (spherical?) area that encircles the metal-enriched disk (or bar), and the radius of this area is $\sim 10^{\prime \prime}$. The most natural explanation - that we see an old, rather metal-poor bulge - is in some contradiction to the known estimates of the size of the bulge of NGC 3623 (18", Hogg et al. 2001; 20", Burkhead \& Hutter 1981). The other interesting feature, two symmetric $\mathrm{H} \beta$ minima at $5^{\prime \prime}-8^{\prime \prime}$ from the center located near the major axis of the isophotes (and of the Mgb isolines), may be a consequence of $\mathrm{H} \beta$ index contamination by gas emission. In this case, we would have detected an ionized-gas torus edge-on; and if so, the circumnuclear chemically decoupled substructure would have a high probability of being a bar.
Since for NGC 3623 we have two sources of data, we have to compare them and to select basic data for quantitative estimates of the stellar population properties. Figures 3 and 4 present this comparison: in Fig. 3 we compare azimuthally averaged index profiles according to the MPFS data and to two independent SAURON data sets, and in Fig. 4 we compare minor-axis index cross-sections simulated over the 2D index maps with the long-slit data of Proctor (2002). As to Fig. 3, the two SAURON data sets agree perfectly despite the very different positions of the galactic nucleus in the micropupil frame and the resulting slightly different spectral response and resolution. The magnesium data of the MPFS and of SAURON show no noticeable discrepancy but the other two indices do: the MPFS $\mathrm{H} \beta$ is lower than the SAURON one by some $0.2-0.3 \AA$, and on the contrary, the SAURON Fe5270 is lower than the MPFS estimates by $0.4-0.5 \AA$. The same shifts were found by us for the index measurements in NGC 3384 (Sil'chenko et al. 2003); and since NGC 3384 was observed with SAURON during the same observational run of March/April 2000, we conclude that these shifts may be systematic. The comparison of the SAURON data with the independently calibrated data of Proctor (2002) in Fig. 4 assures us that the SAURON H $\beta$ 




Fig. 3. The MPFS azimuthally-averaged index profiles for NGC 3623 in comparison with the two sets of the SAURON data.

estimates are correct whereas the Fe5270 estimates in the central part of the galaxy are too low, by the same $0.4-0.5 \AA$. The same conclusions have been made by Sil'chenko et al. (2003) for NGC 3384. Moreover, we have also found a shift of the MPFS $\mathrm{H} \beta$ index profiles in the same sense for a dozen other galaxies in common with the data of Proctor (2002) and/or Fisher et al. (1996) whereas our iron indices are quite consistent with their measurements. Considering all these effects, for safety we take for NGC 3623 the $\mathrm{H} \beta$ estimates from the SAURON data and the $\langle\mathrm{Fe}\rangle$ estimates from the MPFS data to make a quantitative analysis of the luminosity-weighted properties of the stellar population by comparing the observed Lick indices to evolutionary synthesis models.

\subsection{NGC 3627}

Figure 5 presents the index maps obtained for this galaxy with the MPFS. They differ substantially from the index maps of NGC 3623 (Fig. 1). The magnesium map looks rather flat, but the maps of $\mathrm{H} \beta$ and $\langle\mathrm{Fe}\rangle$ do not: $\mathrm{H} \beta$ has an unresolved maximum and $\langle\mathrm{Fe}\rangle$ has an unresolved minimum near the center. Both extrema are shifted by $1^{\prime \prime}-11^{\prime \prime} 5$ to the south-south-west from the continuum peak; as the shifts are identical and the isophotes are slightly distorted, we suggest that perhaps the $\mathrm{H} \beta$ and continuum peaks do not coincide due to a dust effect. So we should treat the substructure indicated by the high $\mathrm{H} \beta$ and low $\langle\mathrm{Fe}\rangle$ as the nucleus. Therefore, inspecting the maps of all three indices together, we can immediately suggest that the nucleus of NGC 3627 is considerably younger than the surrounding bulge and that it is distinguished by an increased magnesium-to-iron ratio.

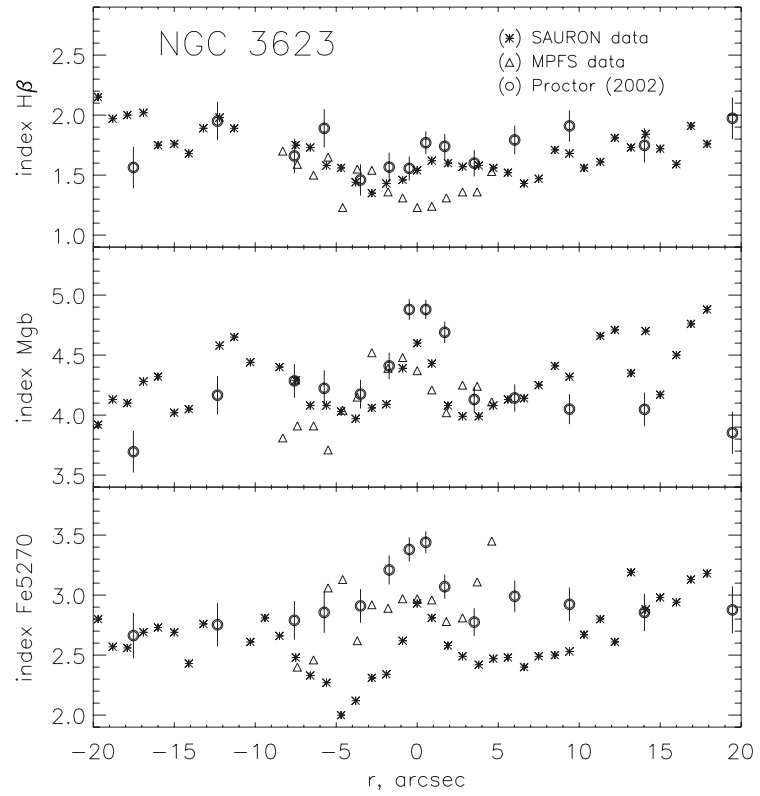

Fig. 4. The minor-axis long-slit index cross-sections by Proctor (2002) for NGC 3623 are plotted in comparison with the minor-axis index profiles simulated from the MPFS 2D index maps and from the SAURON 2D index maps with a digital slit width of $1.25^{\prime \prime}$.

\subsection{Abundances and ages}

Figure 6 presents a $\langle\mathrm{Fe}\rangle$ vs. Mgb diagram. Besides the azimuthally averaged MPFS data for NGC 3623 and NGC 3627, we have also plotted the most modern evolutionary synthesis models for old simple stellar populations by Thomas et al. (2003) calculated for two values of $[\mathrm{Mg} / \mathrm{Fe}], 0.0$ and +0.3 . The observational data points lie mostly between two model sets, so we may conclude that both bulges, that of NGC 3623 and that of NGC 3627 , have $[\mathrm{Mg} / \mathrm{Fe}]=+0.1-+0.2$. The nuclei seem to be slightly off. As for the nucleus of NGC 3623, its displacement toward the locus of $[\mathrm{Mg} / \mathrm{Fe}]=0.0$ may be explained by a lower age; this explanation is favoured by the results of Proctor (2002) for NGC 3623 who, by involving also the Balmer absorption lines of higher orders, has found a minimum of the stellar population age in the nucleus and an age maximum at $R \sim 3^{\prime \prime}$ as well as a flat profile of $[\mathrm{Mg} / \mathrm{Fe}]$ of about +0.15 . But the nucleus of NGC 3627 certainly has $[\mathrm{Mg} / \mathrm{Fe}] \geq+0.3$ implying a brief last event of star formation. As for the total metallicity, NGC 3627 is more metal-poor than NGC 3623. In particular, the nucleus of NGC 3623 is supersolar, $[\mathrm{m} / \mathrm{H}] \geq+0.3$ (Proctor, 2002, gives $[\mathrm{m} / \mathrm{H}] \approx+0.5$ ), and the nucleus of NGC 3627 is subsolar, $[\mathrm{m} / \mathrm{H}] \sim-0.3$. Considering the well-known relation between total luminosity of galaxies and their metallicity, we must note that the close luminosities and morphologies of the two galaxies (NGC 3627 is even slightly more luminous, see Table 1 ) have not made us expect the metallicity difference by a factor of 4 between NGC 3623 and NGC 3627 - such a metallicity difference usually corresponds to a difference of integrated absolute magnitudes of 4 mag.

To determine the ages of the circumnuclear stellar populations in NGC 3623 and NGC 3627, we have to compare 

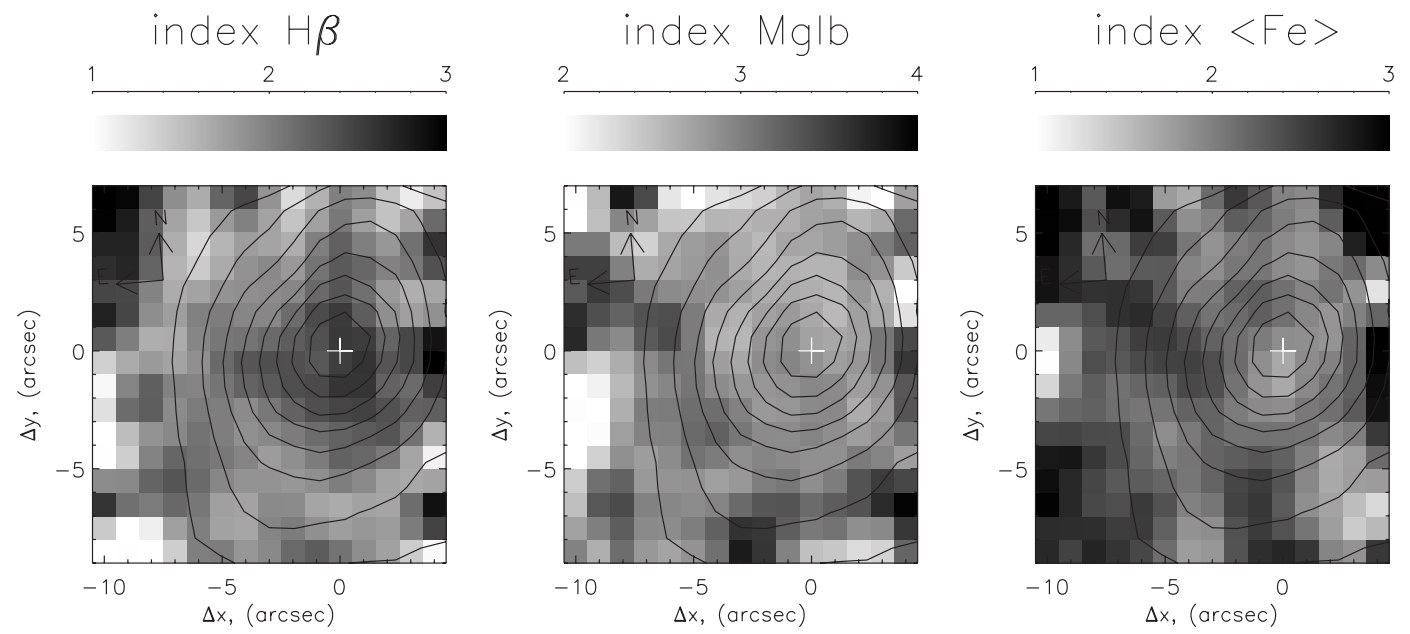

Fig. 5. The MPFS index maps for NGC 3627; $\langle\mathrm{Fe}\rangle \equiv(\mathrm{Fe} 5270+\mathrm{Fe} 5335) / 2$. The green $(\lambda 5000 \AA)$ continuum is overlaid by isophotes.

the stellar hydrogen-line $\mathrm{H} \beta$ index to some metal-line index. Here a severe problem exists in that the Balmer absorption $\mathrm{H} \beta$ is somewhat contaminated by emission. To correct the measured Lick $\mathrm{H} \beta$ index for the emission $\mathrm{H} \beta$ we use the well-known fact that an $\mathrm{H} \alpha$ emission line is always much stronger than $\mathrm{H} \beta$ and an $\mathrm{H} \alpha$ absorption line is always weaker than $\mathrm{H} \beta$, especially in spectra of intermediate-age populations, so the equivalent width of the emission line $\mathrm{H} \alpha$ can be measured more precisely than that of $\mathrm{H} \beta$. In this work we use the results of Ho et al. (1997) for the nuclei of NGC 3623 and NGC 3627, because our efforts to make a Gauss analysis of the red spectra have failed due to the faintness of the $\mathrm{H} \alpha$ emission line in NGC 3623 and to the low stellar velocity dispersion (narrow $\mathrm{H} \alpha$ absorption line) in NGC 3627. Ho et al. (1997) subtracted a pure-absorption-line template from the observed spectra and obtained $E W\left(\mathrm{H} \alpha_{\text {emis }}\right)=0.83 \AA$ for NGC 3623 and $4.25 \AA$ for NGC 3627. For radiative excitation of gas by OB-stars ("H II-region"-type excitation) the wellestablished and lowest possible emission intensity ratio $\mathrm{H} \alpha / \mathrm{H} \beta$ is 2.5-2.7, and it is much larger for shock excitation of the gas. Ho et al. (1997) have classified the nuclear emission spectrum of NGC 3623 as LINER and that of NGC 3627 as transition type between Seyfert 2 and H II. Stasinska \& Sodre (2001) have analysed a large sample of integrated spectra of galaxies of various morphological types and have found a good correlation $E W\left(\mathrm{H} \beta_{\text {emis }}\right)=0.25 E W\left(\mathrm{H} \alpha_{\text {emis }}\right)$; this is just the relation we have to use to calculate $E W\left(\mathrm{H} \beta_{\text {emis }}\right)$, which is in fact the correction of the Lick $\mathrm{H} \beta$ index for the emission. We stress that any substantial contribution of an active nucleus to the gas excitation near the centers of the galaxies or any dust extinction should increase the Balmer decrements and so diminish the correction for the $\mathrm{H} \beta$ emission estimated by our method; therefore the mean stellar ages estimated below using the corrected and the uncorrected $\mathrm{H} \beta$ values are indeed the lower and upper limits of the true luminosity-weighted stellar ages.

Figure 7 presents the age-diagnostic diagrams for our galaxies. For NGC 3623 (Fig. 7 top) we compare $\mathrm{H} \beta$ with Mgb because for this consideration we use the SAURON data, and the SAURON iron index is strongly underestimated (see above). Two models from Thomas et al. (2003) are plotted,

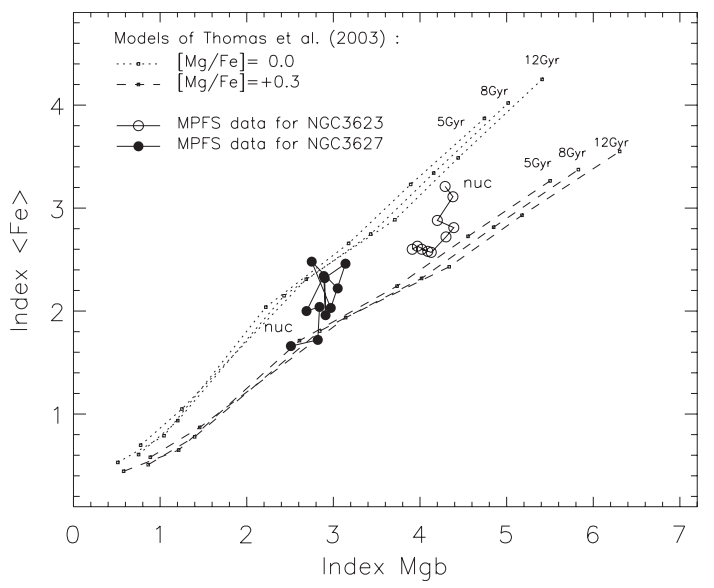

Fig. 6. "Index-index" $\langle\mathrm{Fe}\rangle$ vs. Mgb diagnostic diagram for the azimuthally averaged Lick indices in the center of NGC 3623 and NGC 3627 taken along the radius with a step of 1 " (signs connected by solid lines, the nuclei are marked by "nuc"). The models of Thomas et al. (2003) for $[\mathrm{Mg} / \mathrm{Fe}]=0.0$ and +0.3 are plotted for reference frame; the small signs connected by pointed and dashed lines represent stellar population models of equal ages; the metallicities for the models are $+0.67,+0.35,0.0,-0.33,-1.35$, and -2.25 if one takes the signs from top to bottom.

for $[\mathrm{Mg} / \mathrm{Fe}]=0.0$ and +0.3 , because the abundance ratio in NGC 3623 is somewhere between these values, $+0.1-+0.2$. The azimuthally averaged "bulge" of NGC 3623 is old, $T_{\text {bul }}=$ 10-15 Gyr, and has nearly solar metallicity, whereas the nucleus when corrected for the emission is found to have an intermediate age, $\sim 5 \mathrm{Gyr}$, and a very high metallicity, +0.6 dex. These results are in general agreement with those of Proctor (2002). For NGC 3627 (Fig. 7 bottom) we are able to compare $\mathrm{H} \beta$ with the composite metal index $[\mathrm{MgFe}] \equiv \sqrt{\mathrm{Mgb}\langle\mathrm{Fe}\rangle}$, which eliminates completely the effect of the $\mathrm{Mg} / \mathrm{Fe}$ ratio on age estimation. The nucleus of NGC 3627 looks very young: after applying the correction for the emission to the $\mathrm{H} \beta$ index we obtain $T \approx 1 \mathrm{Gyr}$ and nearly solar metallicity. The bulge is found to have the same metallicity and an intermediate age of the stellar population, 3-5 Gyr. Note that in this age range the possible systematic uncertainty of the $\mathrm{H} \beta$ index by some $0.2 \AA$ 

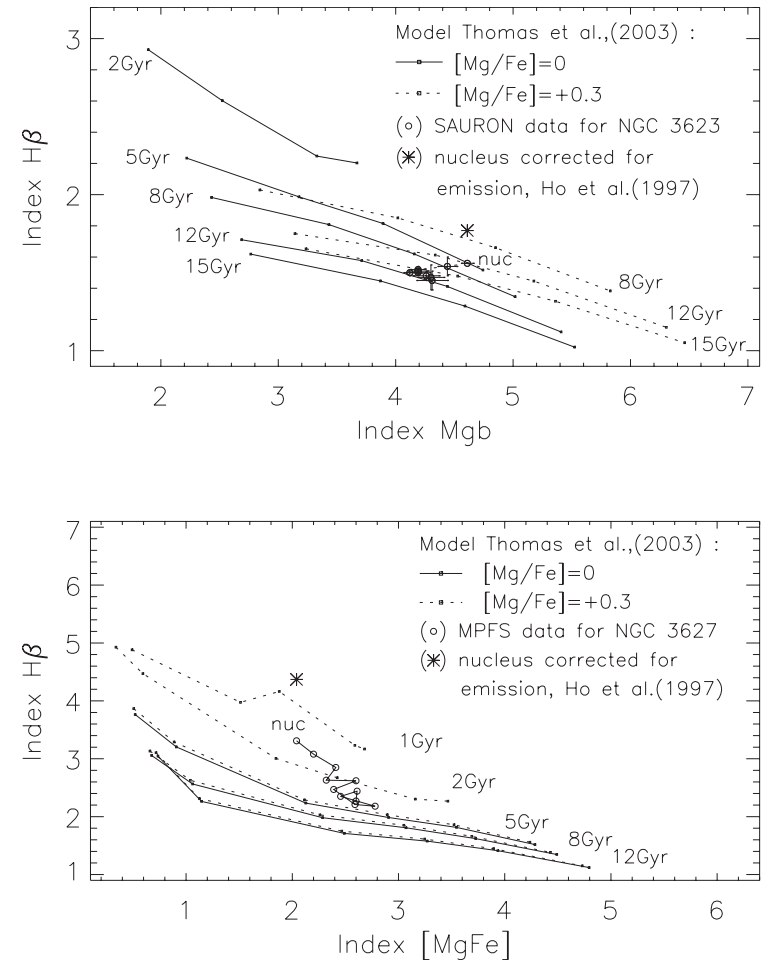

Fig. 7. Age-diagnostics diagrams: $\mathrm{H} \beta$ vs. Mgb for the azimuthally averaged Lick indices in the center of NGC 3623 and $\mathrm{H} \beta$ vs. [MgFe], $[\mathrm{MgFe}] \equiv(\mathrm{Mgb}\langle\mathrm{Fe}\rangle)^{1 / 2}$, for the azimuthally averaged Lick indices in the center of NGC 3627 . The galaxy data - large signs connected by solid lines - are taken along the radius in steps of $\sim 1^{\prime \prime}$; the nuclei are marked "nuc". The $\mathrm{H} \beta$ measurements for the nuclei corrected for the emission contamination as described in the text are also plotted as separate asterisks. The models of Thomas et al. (2003) for $[\mathrm{Mg} / \mathrm{Fe}]=0.0$ and +0.3 are plotted as a reference frame; the small signs connected by solid and dashed lines represent stellar population models of equal ages; the metallicities for the models are $+0.67,+0.35,0.0,-0.33$, -1.35 , and -2.25 , if one takes the signs from right to left.

which may be suspected for the MPFS data has no substantial effect on the age estimates. The main conclusion of this subsection is that the mean stellar population properties in the nuclei of NGC 3623 and NGC 3627 are quite different.

\section{Stellar and gas kinematics in the centers of NGC 3623 and NGC 3627 and their circumnuclear structures}

Since the integral-field spectroscopy provides us with twodimensional line-of-sight velocity fields, we are able now to analyse both geometry of rotation and central structure of the galaxies. If we have an axisymmetric mass distribution and rotation on circular orbits, the direction of the maximum central line-of-sight velocity gradient (we shall call it "kinematical major axis") should coincide with the line of nodes, as should the photometric major axis; whereas in the case of a triaxial potential the isovelocities are aligned with the principal axis of the ellipsoid (see e.g. the simulations of bars by Vauterin \& Dejonghe 1997 and references therein), and generally the kinematical and photometric major axes diverge, showing turns with respect to the line of nodes in opposite senses if the main axis of the triaxial potential is not strictly aligned with the line of nodes (Monnet et al. 1992; Moiseev \& Mustsevoy 2000). In a simple case of cylindric (disk-like) rotation we have a convenient analytical expression for the azimuthal dependence of the central line-of-sight velocity gradients within the area of solid-body rotation:

$\mathrm{d} v_{r} / \mathrm{d} r=\omega \sin i \cos \left(\mathrm{PA}-\mathrm{PA}_{0}\right)$,

where $\omega$ is the deprojected central angular rotation velocity, $i$ is the inclination of the rotation plane, and $\mathrm{PA}_{0}$ is the orientation of the line of nodes, coinciding in the case of an axisymmetric ellipsoid (or a thin disk) with the photometric major axis. So by fitting the azimuthal variations of the central line-of-sight velocity gradients by a cosine law we can determine the orientation of the kinematical major axis by its phase and the central angular rotation velocity by its amplitude. It is our main tool of kinematical analysis.

\subsection{NGC 3623}

de Zeeuw et al. (2002) have already claimed to detect the kinematical signatures of the presence of a compact circumnuclear stellar disk in the center of NGC 3623 from their preliminary analysis of the SAURON data. Let us consider the issue in more detail.

Figure 8 presents the kinematical information on NGC 3623 that we have derived from the green and red MPFS spectra: line-of-sight velocity fields for the stars and ionized gas, the stellar velocity dispersion field, and the [N II] $\lambda 6583$ emission-line intensity distribution for comparison with the orientation of the kinematical major axis of the ionized gas. Both stellar and gas velocity fields within $R \leq 5^{\prime \prime}$ imply regular rotation in the symmetry plane of the galaxy whose line of nodes is at $\mathrm{PA}_{0}=174^{\circ}$ (see Table 1 ). Prominent crowding of the stellar isovelocities near the major axis signifies the presence of a circumnuclear stellar disk; and the intensity distribution of the emission is evidence for a gas confinement to the same circumnuclear disk. However, a little farther from the center the isovelocities, both of the stellar and gaseous components, tend to turn consistently. This turn is much more evident in Fig. 9, left, where the SAURON data combined from two different telescope pointings are presented. Even the stellar isovelocities $\pm 40 \mathrm{~km} \mathrm{~s}^{-1}$ from the systemic velocity, which belong to the circumnuclear disk near the center, certainly turn perpendicular to the north-north-east direction in the outer parts of the field. Beyond $R \approx 8^{\prime \prime}$ we observe practically cylindric rotation around a skewed rotation axis. The appearance of the stellar velocity dispersion map is even more striking (Fig. 9, right). There is a half-sphere of enhanced $\sigma_{*}$, with a radius of $8^{\prime \prime}-10^{\prime \prime}$, and with a hole inside it. The central minimum of the stellar velocity dispersion is also clearly seen in the MPFS data (Fig. 8) though the MPFS stellar velocity dispersion map suggests a more complete ring of enhanced $\sigma_{*}$ closed to the south of the nucleus. We should stress here that the zone of enhanced $\sigma_{*}$ coincides perfectly with the zone of low Mgb index (Fig. 2); both features are quite typical for a bulge. But it must mean 

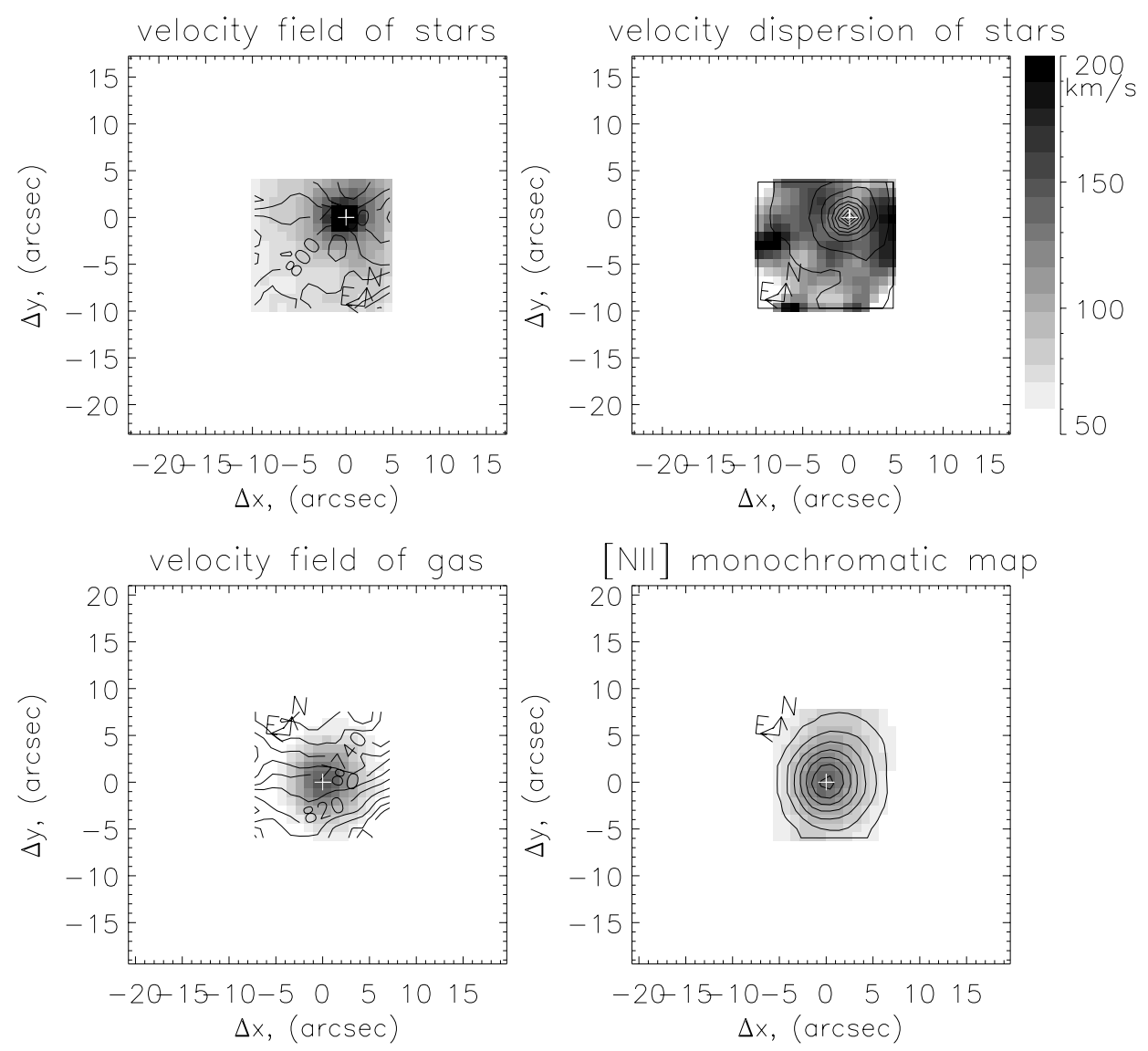

Fig. 8. The line-of-sight velocity fields of the stellar component (top left, isolines) and of the ionized gas (bottom left, isolines), and the stellar velocity dispersion map (top right, gray-scaled) and the [NII] emission line intensity distribution (bottom right, gray-scaled) in the central part of NGC 3623 according to the MPFS data. The continuum intensity is shown as gray-scaled in the left plots and as isophotes in the right plots.
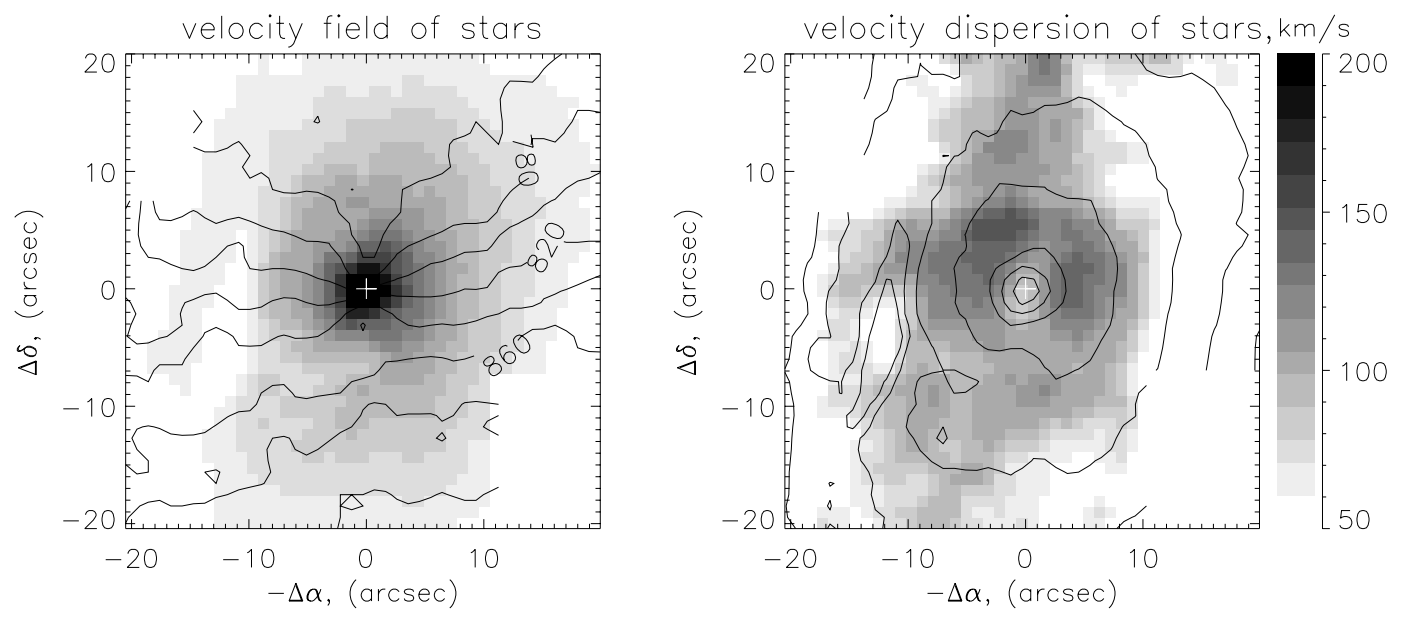

Fig. 9. The line-of-sight velocity field of the stellar component (left, isolines) and the stellar velocity dispersion map (right, gray-scaled) in the central part of NGC 3623 according to the SAURON data. The continuum intensity is shown as gray-scaled in the left plot and as isophotes in the right plot.

again that the bulge of NGC 3623 is confined within $R=10^{\prime \prime}$ whereas from the analysis of photometric profiles it seems to dominate within $R<20^{\prime \prime}$ (Burkhead \& Hutter 1981). To check the bulge extension in NGC 3623 we have undertaken our own analysis of its surface brightness profile using the $K$-band data of the 2MASS survey now available from NED; the results are presented in Fig. 10. Indeed, inside $R=30^{\prime \prime}$ the surface brightness profile can be perfectly fitted by a de Vaucouleurs' law; moreover, the brightness profile of the outer large-scale disk is of Freeman's II-type, that is, shows a depression in the center. The inner truncation of the large-scale stellar disk of NGC 3623 at $R=98^{\prime \prime}$ has also been reported by Baggett et al. (1998); they have found the effective radius of the bulge in the $V$-band to be equal to $69^{\prime \prime}$. We cannot yet understand 


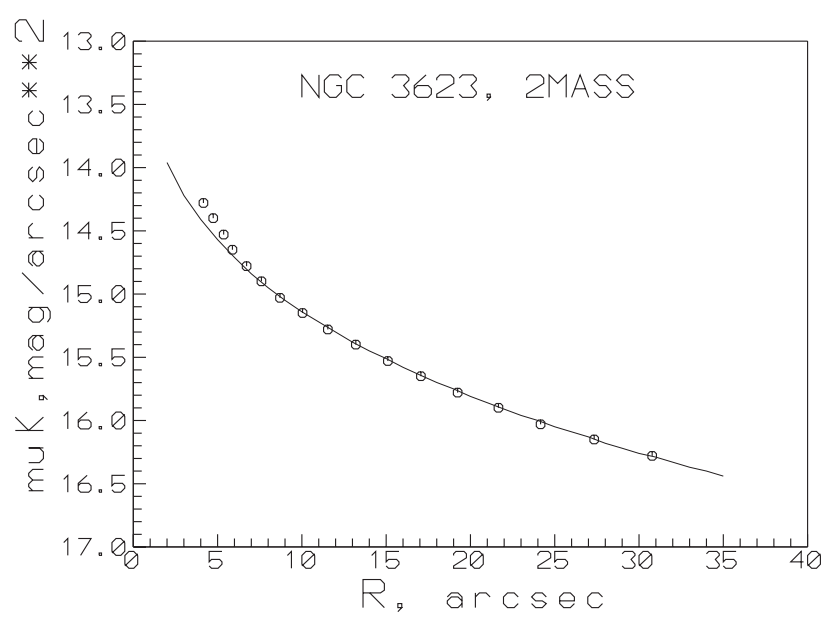

Fig. 10. Fitting of the central azimuthally-averaged $K$-band brightness profile of NGC 3623 by a de Vaucouleurs law. An excess of the brightness within $R<6^{\prime \prime}$ may be attributed to the circumnuclear stellar disk.

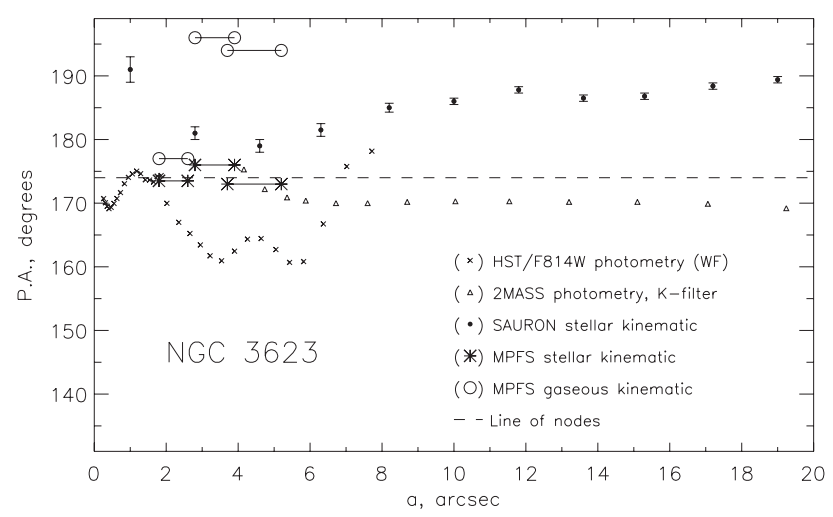

Fig. 11. Isophote major-axis position angle compared to the orientations of the kinematical major axes (see text) for the stars and ionized gas in the center of NGC 3623. The line of nodes determined from the outermost disk isophote orientation, $\mathrm{PA}=174^{\circ}$, is traced by a dashed line.

this discrepance between the photometric size of the bulge and its zone of influence on the dynamics and stellar population properties integrated in the line of sight.

Figures 11 and 12 quantify our impressions about stellar and gaseous kinematics. In Fig. 11 we compare the orientations of the kinematical major axes with those of the isophotes. In the very center, $R \leq 2^{\prime \prime}$, the HST/WFPC2/F814W data reveal the isophote orientation coinciding with the outer line of nodes. The drop of PA at larger $R$ is perhaps an artifact of a dust effect because it is not supported by the low-resolution $\mathrm{K}$-data of the 2MASS survey. The kinematical major axis orientations obtained from the MPFS data by fitting formula (1) to the azimuthal variations of the line-of-sight velocity gradients imply that the stars rotate axisymmetrically in the plane of the circumnuclear disk (now definitely a disk, not a bar) up to $R \approx 6^{\prime \prime}$ whereas the gas, even rather close to the center, $R \geq 3^{\prime \prime}$, shows some signs of non-circular motions. The SAURON stellar velocity field has been analysed by applying the well-known method of tilted rings (Begeman 1989) realized by Dr. A.V. Moiseev under IDL as the software DETKA. The SAURON data reveal a turn of the stellar kinematical

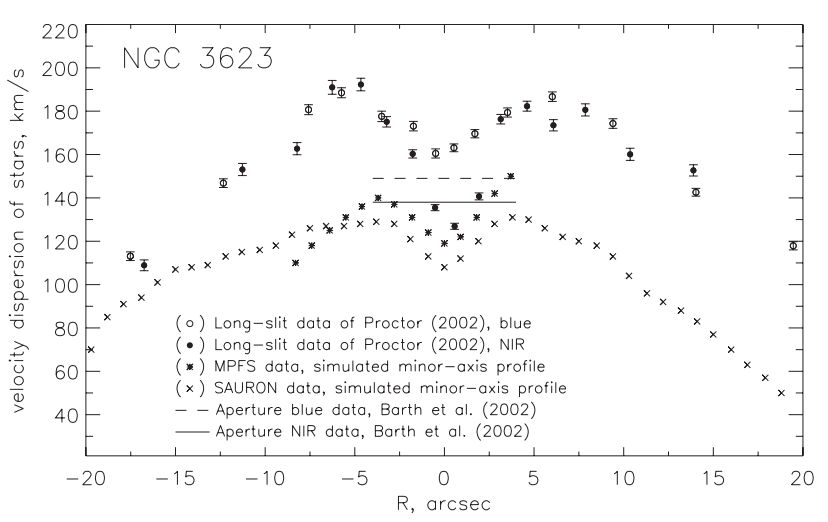

Fig. 12. Comparison of the stellar velocity dispersion profiles simulated along the minor axis using our 2D fields (MPFS and SAURON) for the center of NGC 3623 with the long-slit data of Proctor (2002) and aperture data of Barth et al. (2002). The slit width used in the simulations is $1.25^{\prime \prime}$.

major axis at $R=8^{\prime \prime}-20^{\prime \prime}$ by $+10^{\circ}-+15^{\circ}$ from the line of nodes; as the large bar of NGC 3623 is elongated in PA $\approx 155^{\circ}$, that is, its $\triangle \mathrm{PA} \approx-19^{\circ}$, the whole picture is consistent with the stellar elliptical $\mathrm{x} 1$ orbits within the bar potential. It is an additional proof of what we have already established from the index and $\sigma_{*}$ maps: that at $R>8^{\prime \prime}$ we do not see noticeable influence of the bulge stellar component.

The minimum of the stellar velocity dispersion in the nucleus of NGC 3623 has already been reported by Proctor et al. (2000) and by Proctor (2002) from their long-slit observations along the minor axis of the galaxy. In Fig. 12 we compare the data of Proctor (2002) with the minor-axis cross-sections simulated over the MPFS and SAURON stellar velocity dispersion maps. Although there is a noticeable systematic shift between our measurements and those of Proctor (2002), the qualitative behaviour of the radial profiles of the stellar velocity dispersion is the same: the highest $\sigma_{*}$ is observed at $R=4^{\prime \prime}-6^{\prime \prime}$, and in the nucleus there is a drop of $\sigma_{*}$ by some $20 \%$. It is interesting that according to Proctor (2002), who has measured the stellar velocity dispersion using two different spectral ranges, in the NIR Ca II triplet the drop of $\sigma_{*}$ is deeper and reaches about $30 \%$; it may be an evolutionary effect of probing stars of different ages in different spectral ranges. Of course, we wonder which scale of $\sigma_{*}$ is correct, ours or that of Proctor (2002). In Fig. 12 we have also plotted the quite recent aperture measurements of Barth et al. (2002), also obtained in two spectral ranges. The latter data imply that "the truth is somewhat between" our and Proctor's measurements.

\subsection{NGC 3627}

Figure 13 presents kinematical maps for the center of NGC 3627. Although the spatial resolution of both the green and the red observations is rather low (see Table 2) and we cannot trace any variations of the rotation over the field of view, its total character is clear. The stellar component rotates regularly and axisymmetrically and probably forms a circumnuclear disk or an oblate bulge. As for the ionized gas, its emission brightness distribution is a twin to that in the center of NGC 3623 , 

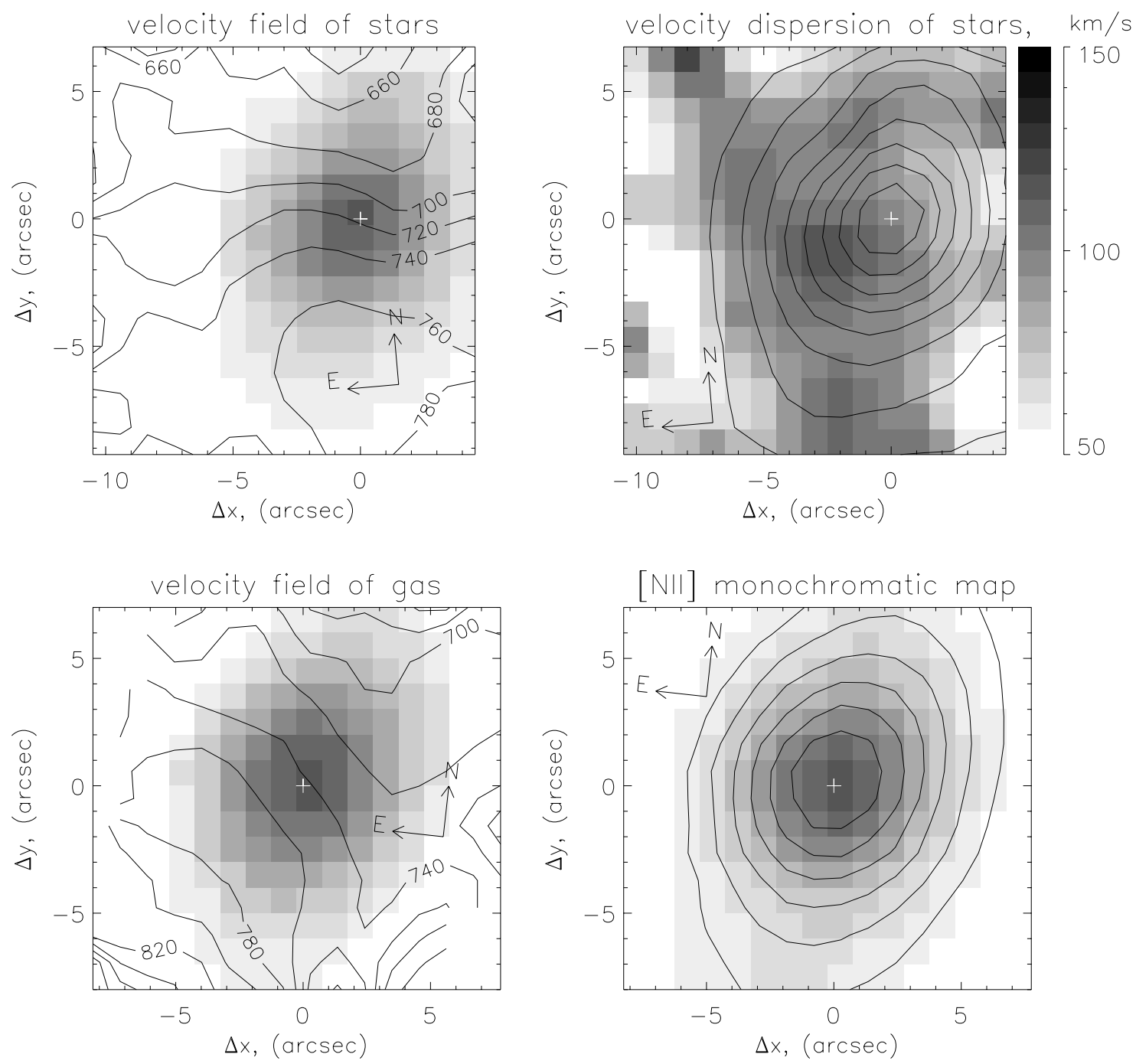

Fig. 13. The line-of-sight velocity fields of the stellar component (top left, isolines) and of the ionized gas (bottom left, isolines), and the stellar velocity dispersion map (top right, gray-scaled) and the [NII] emission line intensity distribution (bottom right, gray-scaled) in the central part of NGC 3627. The continuum intensity is shown as gray-scaled in the left plots and as isophotes in the right plots.

but the velocity field is quite different: all the isovelocities, from the innermost to the outermost ones, are turned as a whole with respect to the kinematical major axis of the stars. Finally, the stellar velocity dispersion distribution shows a peak instead of the hole of NGC 3623; however, this peak does not coincide exactly with the continuum peak, and it is shifted in another direction from the position of the $\mathrm{H} \beta$ index peak.

In Fig. 14 we compare the orientations of the kinematical major axes with the orientation of the NIR (HST/NICMOS) central isophotes. Taking into account the difference of spatial resolution, we may conclude that the stellar rotation follows the orientation of the photometrical major axis, and at $R=$ $2^{\prime \prime}-5^{\prime \prime}$ both are close to the line of nodes of the global disk of NGC 3627. This double coincidence implies an axisymmetry of the central part of the galaxy. The kinematical major axis of the ionized gas deviates by $-35^{\circ}$ to $-40^{\circ}$ from the line of nodes and from the elongation of the emission brightness distribution (see Fig. 13). Since the global bar of NGC 3627 is aligned at $\mathrm{PA} \approx 160^{\circ}$, or deviates from the line of nodes in the same negative sense of $\triangle \mathrm{PA}$, the orientation of the gas kinematical

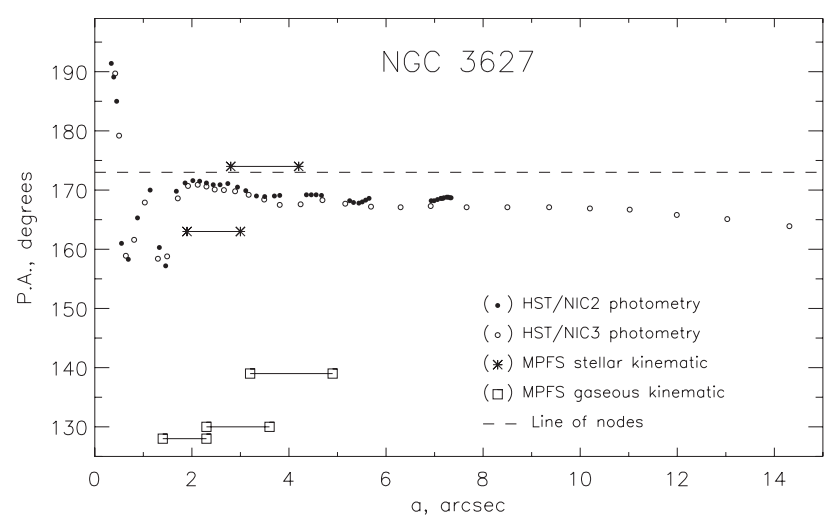

Fig. 14. Isophote major-axis position angle compared to the orientations of the kinematical major axes (see text) for the stars and ionized gas in the center of NGC 3627. The line of nodes determined from the outermost disk orientation, $\mathrm{PA}=173^{\circ}$, is traced by a dashed line.

major axis cannot be explained by elliptical $\mathrm{x} 1$ orbits, as has been done in the case of NGC 3623. Since the morphology 
of the gas and/or dust distribution in the center of NGC 3627 does not imply any regular off-plane structures like disks or rings, the most natural way to explain the gas velocity field of NGC 3627 may be by strong radial motions. If we suppose that the eastern side of the galaxy is the nearest to us by assuming that its spiral arms are trailing, we should conclude that perhaps we deal with gas inflow into the nucleus.

\section{Conclusions and discussion}

Unlike our previous targets, NGC 3384/NGC 3368 in the Leo I Group and NGC 5574/NGC 5576 in LGG 379, NGC 3623 and NGC 3627 do not appear to experience synchronous evolution. The mean ages of their circumnuclear stellar populations are quite different. Since to derive the stellar population properties we have used the model synthesis calculations for Simple Stellar Populations (SSPs), that is, for stellar systems with homogeneous ages and metallicities of stars, the results are somewhat conventional: the real galaxies consist of stars of various ages. However due to fast luminosity evolution of stellar populations our luminosity-weighted mean age estimate must be close to an age of the latest star formation event. The magnesium overabundance of the nucleus in NGC 3627 found here is evidence for a very brief last star formation event $\sim 1$ Gyr ago whereas the central part of NGC 3623 looks quiescent. Moreover, though the global bars of both galaxies have similar morphologies, it is only in the center of NGC 3627 that we observe noticeable irregular, possibly radial motions of the gas, and the stars and the ionized gas in the center of NGC 3623 show more or less stable rotation. However, NGC 3623 has a chemically distinct core - a relic of a circumnuclear star formation burst - which is shaped as a compact, dynamically cold stellar disk with a radius of $\sim 250-350 \mathrm{pc}$ that has been formed not later that 5 Gyr ago.

What may be the reason for these different properties of the central regions in NGC 3623 and NGC 3627? Perhaps just what was guessed by Zwicky (1956) and Vorontsov-Vel'yaminov (1959): the latter is an interacting galaxy and the former is not. Rots (1978) tried to explain a spectacular long plume of NGC 3628 seen both in the optical image and in the $21 \mathrm{~cm}$ neutral hydrogen line and proposed a scenario of tidal interaction with NGC 3627. In the frame of his model NGC 3627, moving along a parabolic orbit, passed through perigalacticon at $25 \mathrm{kpc}$ from NGC 3628 some $8 \times 10^{8} \mathrm{yr}$ ago. Though the numerical simulations were very crude - NGC 3628 consisted of only 441 test particles and NGC 3627 was taken as a single gravitating point, - Rots (1978) obtained a picture quite consistent with the morphology of NGC 3628. He argued that NGC 3627 could not experience a noticeable tidal transformation during this encounter because its orbit was almost retrograde with respect to its intrinsic rotation. However, Zhang et al. (1993) combined high-resolution observations of NGC 3627 in CO and in H I and found certain signatures of violent dynamical evolution of the galaxy after the proposed encounter with NGC 3628. First of all, the outermost southern $\mathrm{H}$ I clouds show visible counterrotation with respect to the more inner gas. But what is even more striking is an enormous gas concentration in the center of the galaxy.
Zhang et al. (1993) detect an inner molecular-gas bar with total mass not less than $4 \times 10^{8} M_{\odot}$; this means that according to their estimates the ISM contributes not less than $30 \%$ to the total dynamical mass within $R=750$ pc. For comparison, within $R=7 \mathrm{kpc}$ the ISM mass constitutes less than $3 \%$ of the dynamical mass. Zhang et al. (1993) conclude that "continuous and efficient radial mass accretion across the entire galactic disk" is unavoidable in NGC 3627. The signatures of this accretion may be directly observed by us in the central gas velocity field as the turn of the kinematical major axis of the gas. Note also that the time passed since the close encounter with NGC 3628 in the simulations by Rots (1978) coincides with the age of the last star formation burst in the nucleus of NGC 3627. Was this burst triggered by the tidal interaction? And the last question inspired by the successful model of Rots (1978): if the whole picture requires a parabolic orbit, was NGC 3627 a member of the Leo Triplet before its encounter with NGC 3628? If not, the visible resemblance between NGC 3623 and NGC 3627 is a simply chance, and we should not expect synchronous evolution of NGC 3623 and NGC 3627 over timescales of several Gyrs.

Another interesting problem relates to the drop in the stellar velocity dispersion in the nucleus of NGC 3623. There are several cases known of stellar velocity dispersion drops in the centers of early-type disk galaxies, and recently Wozniak et al. (2003) have tried to model this phenomenon in the frame of self-consistent N-body simulations including stars, gas, and star formation. Indeed, after $\sim 0.4$ Gyr of numerical evolution their galaxy has developed a central velocity dispersion drop due to nuclear gas inflow, subsequent star formation and the appearance of young luminous stars born from dynamically cold gas. However, the dynamical evolution seems to be rather fast, and after another $0.5 \mathrm{Gyr}$ the central $\sigma_{z}$ of the "new stellar population" increases by a factor of 2 due to the gravitational interaction with the gas and with the old and hotter population. To put this into perspective, Wozniak et al. (2003) note that " $\sigma_{z}$ of the new population is expected to become similar to the $\sigma_{z}$ of the old population in at least 2 Gyr". Let us compare these model timescales to our estimates of ages of the stellar populations in the centers of NGC 3623 and NGC 3627 . NGC 3623 has an age of the nuclear stellar population not less than $5 \mathrm{Gyr}$ and a prominent stellar velocity dispersion drop in the nucleus. Much younger stars dominate in the nucleus of NGC 3627, their $T \sim 1 \mathrm{Gyr}$, and it has only marginal $\sigma_{*}$ depression near the center, according to the long-slit data of Heraudeau \& Simien (1998); in our panoramic data we see not even a marginal $\sigma_{*}$ drop in the center of this galaxy but rather a peak in the stellar velocity dispersion shifted slightly with respect to the photometric center. The relation between the age of the nuclear stellar population and the prominence of the drop in the stellar velocity dispersion obtained by us is in fact opposite to the model predictions by Wozniak et al. (2003). Moreover, recently we found a strong example of a stellar velocity dispersion drop in a galaxy center where the mean stellar age is older than 12 Gyr - it is NGC 2768 (Sil'chenko \& Afanasiev 2004; see also Emsellem et al. 2004). So, though the statistics is rather small yet, no anti-correlation between the prominence of the drop in the stellar velocity dispersion and the age of the stellar 
population in the center of a galaxy is seen. We have to admit that the problem of the origin of the drop in velocity dispersion in the center and of its long life remains unsolved.

Acknowledgements. We thank Dr. A. V. Moiseev of SAO RAS for analysing the stellar velocity field of NGC 3623 with his software DETKA. During the data analysis we have used the LyonMeudon Extragalactic Database (LEDA) supplied by the LEDA team at the CRAL-Observatoire de Lyon (France) and the NASA/IPAC Extragalactic Database (NED) which is operated by the Jet Propulsion Laboratory, California Institute of Technology, under contract with the National Aeronautics and Space Administration. This research is partly based on data taken from the ING Archive of the UK Astronomy Data Centre and on observations made with the NASA/ESA Hubble Space Telescope, obtained from the data archive at the Space Telescope Science Institute, which is operated by the Association of Universities for Research in Astronomy, Inc., under NASA contract NAS 5-26555. The study of nearby galaxy groups is supported by the grant of the Russian Foundation for Basic Researches 04-02-16087 and the study of the evolution of galactic centers - by the Federal Scientific-Technical Program - contract of the Science Ministry of Russia No.40.022.1.1.1101.

\section{References}

Bacon, R., Copin, Y., Monnet, G., et al. 2001, MNRAS, 326, 23

Baggett, W. E., Baggett, S. M., \& Anderson, K. S. J. 1998, AJ, 116, 1626

Barth, A. J., Ho, L. C., \& Sargent, W. L. W. 2002, AJ, 124, 2607

Begeman, K. C. 1989, A\&A, 223, 47

Burkhead, M. S., \& Hutter, D. J. 1981, AJ, 86, 523

Emsellem, E., Cappellari, M., Peletier, R. F., et al. 2004, MNRAS, 352,721

Fisher, D., Franx, M., \& Illingworth, G. 1996, ApJ, 459, 110

Haynes, M. P. 1981, AJ, 86, 1126
Haynes, M. P., Giovanelli, R., \& Roberts, M. S. 1979, ApJ, 229, 83

Heraudeau, P., \& Simien, F. 1998, A\&AS, 133, 317

Ho, L. C., Filippenko, A. V., \& Sargent, W. L. W. 1997, ApJS, 112, 315

Hogg, D. E., Roberts, M. S., Bregman, J. N., \& Haynes, M. P. 2001, AJ, 121, 1336

Moiseev, A. V., \& Mustsevoy, V. V. 2000, Pis'ma v AZh, 26, 657

Monnet, G., Bacon, R., \& Emsellem, E. 1992, A\&A, 253, 366

Paturel, G., Teerikorpi, P., Theureau, G., et al. 2002, A\&A, 389, 19

Proctor, R. N. 2002, Ph.D. Thesis

Proctor, R. N., Sansom, A. E., \& Reid, I. N. 2000, MNRAS, 311, 37

Rots, A. H. 1978, AJ, 83, 219

Sage, L. J. 1993, A\&A, 272, 123

Sil'chenko, O. K. 1997, AZh, 74, 643

Sil'chenko, O. K., \& Afanasiev, V. L. 2004, AJ, 127, 2641

Sil'chenko, O. K., Afanasiev, V. L., Chavushyan, V. H., \& Valdes, J.-R. 2002, ApJ, 577, 668

Sil'chenko, O. K., Moiseev, A. V., Afanasiev, V. L., Chavushyan, V. H., \& Valdes, J.-R. 2003, ApJ, 591, 185

Stasinska, G., \& Sodre, Jr. I. 2001, A\&A, 374, 919

Thomas, D., Maraston, C., \& Bender, R. 2003, MNRAS, 339, 897

Vauterin, P., \& Dejonghe, H. 1997, MNRAS, 286, 812

Vlasyuk, V. V. 1993, Astrofiz. issled. (Izv. SAO RAS), 36, 107

Vorontsov-Vel'yaminov, B. A. 1959, Atlas and Catalogue of Interacting Galaxies, Part I (Moscow Univ. Press)

Whitmore, B. C., Schechter, P. L., \& Kirshner, R. P. 1979, ApJ, 234, 68

Worthey, G. 1994, ApJS, 95, 107

Worthey, G., Faber, S. M., Gonzalez, J. J., \& Burstein, D. 1994, ApJS, 94, 687

Wozniak, H., Combes, F., Emsellem, E., \& Friedli, D. 2003, A\&A, 409, 469

de Zeeuw, P. T., Bureau, M., Emsellem, E., et al. 2002, MNRAS, 329, 513

Zhang, X., Wright, M., \& Alexander, P. 1993, ApJ, 418, 100

Zwicky, F. 1956, Ergeb. Exakten Naturwiss., 29, 344 